\title{
Effect of polypropylene fibres on the compressive and tensile strength of a soft soil, artificially stabilised with binders
}

\author{
António A.S. Correia ${ }^{a,}$ *, Paulo J. Venda Oliveira ${ }^{a}$, Dione G. Custódio ${ }^{b}$ \\ a Department of Civil Engineering, University of Coimbra, R. Luís Reis Santos, 3030-788 Coimbra, Portugal \\ b Quinta das Lameiras, Lt13A, 3500-794 Viseu, Portugal
}

\section{A R T I C L E I N F O}

\section{Article history:}

Received 29 July 2014

Received in revised form

22 November 2014

Accepted 23 November 2014

Available online 18 December 2014

\section{Keywords:}

Geosynthetics

Polypropylene fibres

Compressive strength

Tensile strength

Flexural strength test

Split tensile strength test

\begin{abstract}
A B S T R A C T
This work studies the effect of binder and fibre quantity on the mechanical behaviour of "Baixo Mondego" soft soil, chemically stabilised with binders and reinforced/non-reinforced with short polypropylene fibres. The experimental programme was comprised by four types of tests, one to evaluate compressive strength (unconfined compressive strength test) and three to evaluate tensile strength (direct tensile strength test, split tensile strength test and flexural strength test). The results indicate that increases in binder content cause an increment in the stiffness, the compressive and tensile strength, but have a lower impact on the specimens reinforced with fibres. In general, the addition of a low quantity of fibres to the stabilised soft soil originates a decrease in the stiffness, compressive and direct tensile strength, a reduction of the loss of strength after peak and a change in behaviour, from brittle to more ductile. The results also reveal that the impact of the addition of fibres on the strength depends on the strain mechanism used in each test. Therefore, in flexural strength tests the impact of the fibres is significant, while in the direct tensile strength tests the inclusion of fibres has a negligible effect. Relationships between the compressive and tensile strength are presented at the end of this study, as well as between the tensile strengths evaluated from the direct tensile strength test, split tensile strength test and flexural strength test.
\end{abstract}

(C) 2014 Elsevier Ltd. All rights reserved.

\section{Introduction}

Using the deep mixing method to improve the mechanical characteristics of soft soils ${ }^{1}$ has spread very quickly over the last few decades. Nowadays, this method is used in various countries around the world and its application has become so widespread that it now includes improving: embankments and the foundations of structures, the stability of slopes, the reduction of the vibration of high-speed trains, the mitigation of liquefaction, deep-mixed soil walls (Terashi, 2005; Holm, 2005; Terashi and Kitazume, 2009) and the stabilisation/solidification of contaminated soils (Al-Tabbaa, 2009; Hernandez-Martinez et al., 2009), among others.

\footnotetext{
* Corresponding author. Tel.: +351 239797 277; fax: +351 239797123.

E-mail addresses: aalberto@dec.uc.pt (A.A.S. Correia), pjvo@dec.uc.pt (P.J. Venda Oliveira), dionecustodio@hotmail.com (D.G. Custódio).

1 A soft soil is normally characterized by a high water content, low permeability and usually exhibits low strength $\left(c_{\mathrm{u}}<50 \mathrm{kPa}\right)$ and high compressibility (Hight et al., 1987).
}

In general, cement-based stabilised soils show a good compressive strength but poor tensile and flexural strength and a brittle behaviour (Sukontasukkul and Jamsawang, 2012). When the stabilised soil is subjected to seismic loads, either horizontal displacements (as in the case of columns installed under the sides of embankments and in slopes) or lateral earth pressures (as for deep-mixed soil walls), the material needs to have some flexural strength to withstand the bending moment applied, otherwise the stabilised material tends to fail under tension, due to its brittleness (Sukontasukkul and Jamsawang, 2012). When deep mixing columns (DMCs) are used in excavated walls, these columns are usually reinforced by steel $\mathrm{H}$ beams in order to obtain the required flexural strength to withstand the lateral earth pressures. An alternative method to improve the flexural and tensile behaviour is to add short fibres into the soilbinder-water mixture, as is usually used in concrete.

Various studies have been published about the effect of synthetic fibres on the behaviour of stabilised soils, fundamentally based on the results of unconfined compressive strength tests (Kaniraj and Havanagi, 2001; Cai et al., 2006; Khattak and Alrashidi, 2006; Tang et al., 2007; Consoli et al., 2010, 2011a, 2012, 2013a, 2013b, 2013c; 
Park, 2009, 2011; Estabragh et al., 2012; Olgun, 2013) and split tensile strength tests (Khattak and Alrashidi, 2006; Consoli et al., 2011b, 2012, 2013a, 2013b, 2013c; Olgun, 2013). A few authors have studied this theme based on triaxial tests (Consoli et al., 2009; Hamidi and Hooresfand, 2013), direct shear tests (Cai et al., 2006; Tang et al., 2007), flexural tests (Sukontasukkul and Jamsawang, 2012), isotropic compression tests (Santos et al., 2010) and cyclic shear tests (Chauhan et al., 2008; Festugato et al., 2013). In general, these studies have been performed with sandy or clayey soils stabilised with a low binder content (less than about 10\%), and they have shown that an increment in the amount of fibres induces: increases in compressive and tensile strength (Kaniraj and Havanagi, 2001; Cai et al., 2006; Khattak and Alrashidi, 2006; Tang et al., 2007; Consoli et al., 2010, 2011a; Park, 2011; Estabragh et al., 2012; Olgun, 2013), increases in residual strength (Tang et al., 2007; Sukontasukkul and Jamsawang, 2012), reductions in stiffness (Tang et al., 2007) and changes the behaviour of the stabilised soil from a brittle one to a more ductile one (Tang et al., 2007; Estabragh et al., 2012). However, Consoli et al. (2009) showed that the reinforcing effect of polypropylene fibres becomes less effective for high cement contents for a stabilised sandy soil, i.e., the peak strength only increases until $4 \%$ of cement content and after that the effect of the fibre reinforcement has the opposite effect. In fact, when the cement content is high, the stiffness of the stabilised soil is fundamentally governed by the high stiffness of the cement and the polypropylene fibres are, therefore, unable to mobilize the tensile strength before peak failure, since the deformations reached are very low. Thus, increasing the amount of fibres for high cement contents does not have repercussions on the increase of the strength (Consoli et al., 2009). A similar behaviour was also reported by Kaniraj and Havanaji (2001) when studying the indirect tensile behaviour of high plasticity clays stabilized with $10 \%$ of cement and reinforced with a fibre amount higher than $0.3 \%$. On the other hand, Sukontasukkul and Jamsawang (2012), using a stabilised soft soil with a cement content of $10-20 \%$, obtained a slight increase in the flexural strength and a significant increment in the residual strength when the fibres were added to the soil-cement paste.

When soft soils are stabilised with binders, a high binder content is usually required to obtain a suitable strength for the stabilised material. The scientific community has largely ignored the effect of fibres in soft soils stabilised with a high binder content probably because the stabilised soil becomes very stiff with such amounts of binder, which, in theory, is not suitable for reinforcement with polypropylene fibres.

\section{Scope of present study}

This work is intended to make a contribution to the study of the compressive and tensile behaviour of stabilised soft soils with a high binder content and reinforced with short polypropylene fibres. To this end, four types of tests were carried out: unconfined compressive strength (UCS) tests, split tensile strength (STS) tests, direct tensile strength (DTS) tests and flexural strength (FS) tests. The study focuses on the analysis of the effect of the binder quantity both with and without fibres and the effect of the fibre quantity on the compressive and tensile behaviour of the composite material. Additionally, relationships between the compressive strength and the tensile strength are addressed, as well as between the tensile strength evaluated from the STS, DTS and FS tests.

\section{Description of the experimental work}

\subsection{Characteristics of the soft soil}

This study was carried out using a Portuguese soft soil, from the "Baixo Mondego" area, located in the centre of Portugal. The soil was collected from one site and depth $(2.5 \mathrm{~m})$, with the purpose of reducing the soil's natural inherent variability (Coelho, 2000; Venda Oliveira et al., 2010). The natural soil has a predominantly silty grain size distribution ( sand $=14 \%$; silt $=61 \%$; clay $=25 \%$ ), a low unit weight $\left(14.6 \mathrm{kN} / \mathrm{m}^{3}\right)$, a high void ratio $(>2.0)$ and a high natural water content (80.9\%). The soil exhibits a high organic matter content (13.1\%), which affects its general behaviour by inducing a low undrained shear strength $(<25 \mathrm{kPa})$ and a high compressibility and plasticity $\left(w_{\mathrm{L}}=68.0 \% ; w_{\mathrm{P}}=41.9 \%\right)$ (Coelho, 2000; Venda Oliveira et al., 2010; Correia, 2011; Custódio, 2013). This clayey-silt organic soil with a high plasticity is classified by USCS as OH (ASTM D 2487, 1998). The chemical composition of the soil reveals a high content of silica $\left(\mathrm{SiO}_{2}=62 \%\right)$ and alumina $\left(\mathrm{Al}_{2} \mathrm{O}_{3}=16 \%\right)$, which confers pozzolanic properties to the soil (Janz and Johansson, 2002). The soil exhibits a reduced pH value (3.5), which can restrain and/or delay some reactions during the chemical stabilization (Edil and Staab, 2005; Correia, 2011; Kitazume and Terashi, 2013).

\subsection{Characteristics of the binders}

The soft soil was chemically stabilised with a binder mixture composed by Portland cement Type I 42.5 R (EN 197-1, 2000) and blast furnace granulated slag, with a dry weight proportion of 75/ 25 , which was defined in a laboratory optimization study (Correia, 2011). The main characteristics of the binders are presented in Table 1. The Portland cement reacts spontaneously with water (hydraulic material) producing a high quantity of reaction products in a short time. The high content of silica $\left(\mathrm{SiO}_{2}=38.7 \%\right)$ and alumina $\left(\mathrm{Al}_{2} \mathrm{O}_{3}=11.6 \%\right)$ that exists in the slag potentiates the development of the pozzolanic reactions, which are responsible for improving the mechanical properties in the long-term (Taylor, 1997; Janz and Johansson, 2002).

\subsection{Characteristics of the polypropylene fibres}

The polypropylene fibres used in this study are $12 \mathrm{~mm}$ in length, $32 \mu \mathrm{m}$ in diameter and exhibit: a great flexibility, a high specific surface $\left(134 \mathrm{~m}^{2} / \mathrm{kg}\right)$, a density of $905 \mathrm{~kg} / \mathrm{m}^{3}$, a tensile strength of $250 \mathrm{~N} / \mathrm{mm}^{2}$ and a Young's modulus of $3500-3900 \mathrm{~N} / \mathrm{mm}^{2}$ (in accordance with the product datasheet supplied by the manufacturer).

\subsection{Specimen preparation and testing}

Remoulded samples were used to reduce the variability of the natural soil, without changing its mineralogical and granulometric composition. The samples of stabilized soft soil with fibres were prepared in accordance with EuroSoilStab (2001) with the modifications proposed by Correia (2011), described in the following steps: (i) the specified amount of binder was mixed with the quantity of distilled water necessary to increase the natural water content of the soil to $113 \%$, producing a slurry (the water content

Table 1

Composition and specific surface of the binders.

\begin{tabular}{lcc}
\hline Property & Binder & \\
\cline { 2 - 3 } & Portland Cement I 42.5R & Slag \\
\hline $\mathrm{CaO}(\%)$ & 63.02 & 37.02 \\
$\mathrm{SiO}_{2}(\%)$ & 19.70 & 38.74 \\
$\mathrm{Al}_{2} \mathrm{O}_{3}(\%)$ & 5.23 & 11.59 \\
$\mathrm{Fe}_{2} \mathrm{O}_{3}(\%)$ & 2.99 & 0.85 \\
$\mathrm{MgO}(\%)$ & 2.38 & 6.75 \\
Specific surface, $\mathrm{S}\left(\mathrm{m}^{2} / \mathrm{kg}\right)$ & 321.5 & 363.0 \\
\hline
\end{tabular}


increase was defined by Correia (2011) in order to ensure the best compromise between the strength and quality of the samples); (ii) the destructured soft soil, the slurry and fibres were thoroughly mixed using a mechanical mixer at a speed of $142 \mathrm{rpm}$ for $4 \mathrm{~min}$, in order to obtain a homogeneous soil-water-binder-fibre paste; (iii) this paste was introduced and compacted directly into specific moulds for each test (cylindrical, $70 \mathrm{~mm}$ diameter PVC moulds for the UCS, STS and DTS tests; metallic, $250 \times 70 \times 70 \mathrm{~mm}$ parallelepiped moulds for the FS tests) in 2 layers (the STS and FS tests) or 3 layers (the UCS and DTS tests); (iv) each layer was lightly tapped by hand, subjected to vibrations by the application of a hand drill with a steel bar near the inner surface of the mould, and compacted with $100 \mathrm{kPa}$ for $10 \mathrm{~s}$; $(v)$ the surface was lightly scarified and another layer was introduced; ( $v i$ ) the samples were cured for 28 days inside a room with both the temperature $\left(20 \pm 2{ }^{\circ} \mathrm{C}\right)$ and humidity (95 $\pm 5 \%$ ) controlled; (vii) the samples were removed from the moulds after the curing period; (viii) both surfaces of the cylindrical samples were trimmed in order to obtain specimens of a height of $140 \mathrm{~mm}$ (the UCS and DTS tests) or $70 \mathrm{~mm}$ (the STS tests); (ix) the electronic devices (load cell and strain gauge transducer) were set up and adjusted; $(x)$ finally, the tests could start and the data were recorded by an automatic data acquisition system; all the tests were carried out under a constant strain rate of $1 \% / \mathrm{min}$.

All the tests were repeated at least twice, in order to guarantee the reliability of the work. Therefore, in general, for each aspect studied (binder quantity and fibre content), a minimum of eight samples were prepared to carry out two UCS tests, two STS tests, two DTS tests and two FS tests.
Three sets of tests were performed in this work: (i) the first set analyses the effect of the binder quantity (BQ), for four different amounts $\left(125,250,375\right.$ and $\left.500 \mathrm{~kg} / \mathrm{m}^{3}\right)$, on the stabilised soil without fibres (Table 2); (ii) the second set studies the effect of the BQ $\left(125,250,375\right.$ and $\left.500 \mathrm{~kg} / \mathrm{m}^{3}\right)$ on the stabilised soil reinforced with a constant quantity of fibres, $50 \mathrm{~kg} / \mathrm{m}^{3}$ (Table 3); (iii) and in the third set of tests, the effect of the fibre quantity (FQ) is analysed for five different amounts $\left(0,25,50,75\right.$ and $\left.100 \mathrm{~kg} / \mathrm{m}^{3}\right)$ for a constant BQ of $375 \mathrm{~kg} / \mathrm{m}^{3}$ (Table 4). For the first two sets of tests (studying the effect of BQ), the DTS and FS tests were carried out only for a BQ of 375 and $500 \mathrm{~kg} / \mathrm{m}^{3}$. The first set of tests (without fibres) was intended to establish reference values, thus allowing the evaluation of the effect of the addition of fibres on the mechanical properties (strength and stiffness) of the reinforced stabilized soil. Tables 2-4 present the three sets of tests carried out, as well as a summary of the main results obtained.

\section{Analysis of results}

\subsection{Effect of binder quantity}

Fig. 1, Tables 2 and 3 show the effect of the binder quantity without and with fibres $\left(\mathrm{FQ}=50 \mathrm{~kg} / \mathrm{m}^{3}\right)$, in terms of the stressstrain or load-displacement behaviour evaluated from the UCS, STS, DTS and FS tests. It may be seen that with the increment of BQ there is a slight increase in the unit weight as a consequence of the production of a greater amount of cementitious products. As expected, the increment of the binder quantity (BQ) or binder content

Table 2

Effect of the binder quantity - soil stabilised without fibres (Custódio, 2013).

\begin{tabular}{|c|c|c|c|c|c|c|c|c|c|}
\hline \multirow[t]{3}{*}{ Type of test } & \multirow[t]{3}{*}{ Property } & \multicolumn{8}{|c|}{ Binder quantity $\left(\mathrm{kg} / \mathrm{m}^{3}\right)$} \\
\hline & & \multicolumn{2}{|l|}{125} & \multicolumn{2}{|l|}{250} & \multicolumn{2}{|l|}{375} & \multicolumn{2}{|l|}{500} \\
\hline & & $\mathrm{T}_{1}$ & $\mathrm{~T}_{2}$ & $\mathrm{~T}_{1}$ & $\mathrm{~T}_{2}$ & $\mathrm{~T}_{1}$ & $\mathrm{~T}_{2}$ & $\mathrm{~T}_{1}$ & $\mathrm{~T}_{2}$ \\
\hline & Fibre quantity $\left(\mathrm{kg} / \mathrm{m}^{3}\right)$ & 0 & & 0 & & 0 & & 0 & \\
\hline & Binder content, $\mathrm{a}_{\mathrm{w}}(\%)$ & 17.9 & & 35.7 & & 53.6 & & 71.5 & \\
\hline \multirow[t]{4}{*}{ UCS test } & Unconfined compressive strength, $\mathrm{q}_{\mathrm{u}}(\mathrm{kPa})$ & $\begin{array}{l}196.1 \\
{[209.2]}\end{array}$ & 222.3 & $\begin{array}{l}1531.1 \\
{[1566 . .0]}\end{array}$ & 1600.9 & $\begin{array}{l}2761.0 \\
{[2623.4]}\end{array}$ & 2485.9 & $\begin{array}{l}3348.7 \\
{[3226.6]}\end{array}$ & 3104.5 \\
\hline & Deformability modulus for $0.5 \mathrm{q}_{\mathrm{u}}, \mathrm{E}_{\mathrm{u} 50}(\mathrm{MPa})$ & $\begin{array}{l}27.7 \\
{[31.8]}\end{array}$ & 35.9 & $\begin{array}{l}139.7 \\
{[151.9]}\end{array}$ & 164.0 & $\begin{array}{l}193.5 \\
{[190.3]}\end{array}$ & 187.1 & $\begin{array}{l}425.6 \\
{[394.5]}\end{array}$ & 363.4 \\
\hline & Unit weight, $\gamma\left(\mathrm{kN} / \mathrm{m}^{3}\right)$ & $\begin{array}{l}14.50 \\
{[14.51]}\end{array}$ & 14.51 & $\begin{array}{l}14.69 \\
{[14.70]}\end{array}$ & 14.71 & $\begin{array}{l}15.43 \\
{[15.46]}\end{array}$ & 15.49 & $\begin{array}{l}15.50 \\
{[15.55]}\end{array}$ & 15.59 \\
\hline & Final water content, $\mathrm{w}_{\mathrm{f}}(\%)$ & $\begin{array}{l}94.0 \\
{[94.1]}\end{array}$ & 94.3 & $\begin{array}{l}80.0 \\
{[80.0]}\end{array}$ & 80.0 & $\begin{array}{l}68.7 \\
{[68.7]}\end{array}$ & 68.7 & $\begin{array}{l}58.9 \\
{[59.4]}\end{array}$ & 59.9 \\
\hline \multirow[t]{4}{*}{ STS test } & Split tensile strength, $\mathrm{f}_{\mathrm{ct}-\mathrm{ST}}(\mathrm{kPa})$ & $\begin{array}{l}46.5 \\
{[46.5]}\end{array}$ & 46.5 & $\begin{array}{l}249.3 \\
{[261.0]}\end{array}$ & 272.7 & $\begin{array}{l}342.2 \\
{[340.4]}\end{array}$ & 338.5 & $\begin{array}{l}582.3 \\
{[563.7]}\end{array}$ & 545.1 \\
\hline & Vertical displacement at failure, $\delta_{\mathrm{v}}(\mathrm{mm})$ & $\begin{array}{l}2.23 \\
{[2.24]}\end{array}$ & 2.24 & $\begin{array}{l}1.35 \\
{[1.37]}\end{array}$ & 1.48 & $\begin{array}{l}1.12 \\
{[1.17]}\end{array}$ & 1.22 & $\begin{array}{l}0.85 \\
{[0.85]}\end{array}$ & 0.85 \\
\hline & Unit weight, $\gamma\left(\mathrm{kN} / \mathrm{m}^{3}\right)$ & $\begin{array}{l}14.59 \\
{[14.59]}\end{array}$ & 14.59 & $\begin{array}{l}14.94 \\
{[14.96]}\end{array}$ & 14.98 & $\begin{array}{l}15.32 \\
{[15.27]}\end{array}$ & 15.22 & $\begin{array}{l}15.86 \\
{[15.71]}\end{array}$ & 15.56 \\
\hline & Final water content, $\mathrm{w}_{\mathrm{f}}(\%)$ & $\begin{array}{l}90.6 \\
{[90.7]}\end{array}$ & 90.8 & $\begin{array}{l}77.9 \\
{[78.2]}\end{array}$ & 78.4 & $\begin{array}{l}67.2 \\
{[67.1]}\end{array}$ & 67.0 & $\begin{array}{l}58.8 \\
{[58.8]}\end{array}$ & 58.7 \\
\hline \multirow[t]{4}{*}{ DTS test } & Direct tensile strength, $\mathrm{f}_{\mathrm{ct}-\mathrm{DT}}(\mathrm{kPa})$ & $(*)$ & & $(*)$ & & $\begin{array}{l}411.2 \\
{[378.6]}\end{array}$ & 345.9 & $\begin{array}{l}539.3 \\
{[527.8]}\end{array}$ & 516.2 \\
\hline & Deformability modulus for $0.5 \mathrm{f}_{\mathrm{ct}-\mathrm{DT}}, \mathrm{E}_{\mathrm{u} 50}(\mathrm{MPa})$ & & & & & $\begin{array}{l}1413.6 \\
{[1534.4]}\end{array}$ & 1655.3 & $\begin{array}{l}1620.4 \\
{[1644.9]}\end{array}$ & 1669.4 \\
\hline & Unit weight, $\gamma\left(\mathrm{kN} / \mathrm{m}^{3}\right)$ & & & & & $\begin{array}{l}15.45 \\
{[15.44]}\end{array}$ & 15.43 & $(* *)$ & $(* *)$ \\
\hline & Final water content, $\mathrm{w}_{\mathrm{f}}(\%)$ & & & & & $\begin{array}{l}70.7 \\
{[70.4]}\end{array}$ & 70.0 & $\begin{array}{l}59.9 \\
{[60.2]}\end{array}$ & 60.5 \\
\hline \multirow[t]{4}{*}{ FS test } & Flexural strength, $\mathrm{f}_{\mathrm{cf}-\mathrm{F}}(\mathrm{kPa})$ & $(*)$ & & $(*)$ & & $\begin{array}{l}760.5 \\
{[774.0]}\end{array}$ & 787.5 & $\begin{array}{l}1170.0 \\
{[1262.2]}\end{array}$ & 1354.4 \\
\hline & Deflection, $\delta_{\mathrm{v}}(\mathrm{mm})$ & & & & & $\begin{array}{l}0.09 \\
{[0.10]}\end{array}$ & 0.11 & $\begin{array}{l}0.09 \\
{[0.11]}\end{array}$ & 0.12 \\
\hline & Unit weight, $\gamma\left(\mathrm{kN} / \mathrm{m}^{3}\right)$ & & & & & $\begin{array}{l}15.11 \\
{[15.04]}\end{array}$ & 14.97 & $\begin{array}{l}15.50 \\
{[15.46]}\end{array}$ & 15.41 \\
\hline & Final water content, $\mathrm{w}_{\mathrm{f}}(\%)$ & & & & & $\begin{array}{l}66.2 \\
{[66.1]}\end{array}$ & 66.0 & $\begin{array}{l}60.4 \\
{[59.9]}\end{array}$ & 59.4 \\
\hline
\end{tabular}

[...]-Average value; $\left({ }^{*}\right)$ Test not carried out; $\left({ }^{* *}\right)$ Data lost; $\mathrm{T}_{\mathrm{i}}-$ Test $\mathrm{i}$. 
Table 3

Effect of the binder quantity - soil stabilised and reinforced with a constant quantity of fibres of $50 \mathrm{~kg} / \mathrm{m}^{3}$ (Custódio, 2013).

\begin{tabular}{|c|c|c|c|c|c|c|c|c|c|}
\hline \multirow[t]{3}{*}{ Type of test } & \multirow[t]{3}{*}{ Property } & \multicolumn{8}{|c|}{ Binder quantity $\left(\mathrm{kg} / \mathrm{m}^{3}\right)$} \\
\hline & & \multicolumn{2}{|l|}{125} & \multicolumn{2}{|l|}{250} & \multicolumn{2}{|l|}{375} & \multicolumn{2}{|l|}{500} \\
\hline & & $\mathrm{T}_{1}$ & $\mathrm{~T}_{2}$ & $\mathrm{~T}_{1}$ & $\mathrm{~T}_{2}$ & $\mathrm{~T}_{1}$ & $\mathrm{~T}_{2}$ & $\mathrm{~T}_{1}$ & $\mathrm{~T}_{2}$ \\
\hline & Fibre quantity $\left(\mathrm{kg} / \mathrm{m}^{3}\right)$ & 50 & & 50 & & 50 & & 50 & \\
\hline & Binder content, $\mathrm{a}_{\mathrm{w}}(\%)$ & 17.9 & & 35.7 & & 53.6 & & 71.5 & \\
\hline \multirow[t]{4}{*}{ UCS test } & Unconfined compressive strength, $\mathrm{q}_{\mathrm{u}}(\mathrm{kPa})$ & $\begin{array}{l}487.3 \\
{[449.7]}\end{array}$ & 412.1 & $\begin{array}{l}1628.3 \\
{[1589.8]}\end{array}$ & 1551.4 & $\begin{array}{l}2173.0 \\
{[2111.0]}\end{array}$ & 2049.0 & $\begin{array}{l}2929.6 \\
{[3033.3]}\end{array}$ & 3137.0 \\
\hline & Deformability modulus for $0.5 \mathrm{q}_{\mathrm{u}}, \mathrm{E}_{\mathrm{u} 50}(\mathrm{MPa})$ & $\begin{array}{l}24.2 \\
{[24.5]}\end{array}$ & 24.9 & $\begin{array}{l}151.4 \\
{[132.2]}\end{array}$ & 113.1 & $\begin{array}{l}231.0 \\
{[212.4]}\end{array}$ & 193.7 & $\begin{array}{l}267.1 \\
{[237.0]}\end{array}$ & 206.8 \\
\hline & Unit weight, $\gamma\left(\mathrm{kN} / \mathrm{m}^{3}\right)$ & $\begin{array}{l}14.49 \\
{[14.47]}\end{array}$ & 14.44 & $\begin{array}{l}14.96 \\
{[14.85]}\end{array}$ & 14.73 & $\begin{array}{l}15.45 \\
{[15.44]}\end{array}$ & 15.43 & $\begin{array}{l}15.83 \\
{[15.85]}\end{array}$ & 15.87 \\
\hline & Final water content, $\mathrm{w}_{\mathrm{f}}(\%)$ & $\begin{array}{l}85.8 \\
{[88.5]}\end{array}$ & 91.3 & $\begin{array}{l}75.9 \\
{[76.6]}\end{array}$ & 77.2 & $\begin{array}{l}70.0 \\
{[70.5]}\end{array}$ & 71.1 & $\begin{array}{l}59.7 \\
{[59.2]}\end{array}$ & 58.7 \\
\hline \multirow[t]{4}{*}{ STS test } & Split tensile strength, $\mathrm{f}_{\mathrm{ct}-\mathrm{ST}}(\mathrm{kPa})$ & $\begin{array}{l}118.8 \\
{[109.8]}\end{array}$ & 100.8 & $\begin{array}{l}448.6 \\
{[442.1]}\end{array}$ & 435.7 & $\begin{array}{l}353.3 \\
{[404.1]}\end{array}$ & 454.8 & $\begin{array}{l}676.9 \\
{[637.2]}\end{array}$ & 597.5 \\
\hline & Vertical displacement at failure, $\delta_{\mathrm{v}}(\mathrm{mm})$ & $\begin{array}{l}12.59 \\
{[12.70]}\end{array}$ & 12.80 & $\begin{array}{l}4.94 \\
{[8.76]}\end{array}$ & 12.58 & $\begin{array}{l}3.81 \\
{[4.88]}\end{array}$ & 5.95 & $\begin{array}{l}3.52 \\
{[4.04]}\end{array}$ & 4.56 \\
\hline & Unit weight, $\gamma\left(\mathrm{kN} / \mathrm{m}^{3}\right)$ & $\begin{array}{l}14.36 \\
{[14.42]}\end{array}$ & 14.47 & $\begin{array}{l}14.90 \\
{[14.88]}\end{array}$ & 14.85 & $\begin{array}{l}15.19 \\
{[15.43]}\end{array}$ & 15.67 & $\begin{array}{l}15.70 \\
{[15.54]}\end{array}$ & 15.37 \\
\hline & Final water content, $\mathrm{w}_{\mathrm{f}}(\%)$ & $\begin{array}{l}87.5 \\
{[89.3]}\end{array}$ & 91.1 & $\begin{array}{l}65.1 \\
{[64.5]}\end{array}$ & 64.0 & $\begin{array}{l}68.1 \\
{[68.2]}\end{array}$ & 68.4 & $\begin{array}{l}54.9 \\
{[55.2]}\end{array}$ & 55.5 \\
\hline \multirow[t]{4}{*}{ DTS test } & Direct tensile strength, $\mathrm{f}_{\mathrm{ct}-\mathrm{DT}}(\mathrm{kPa})$ & $(*)$ & & $(*)$ & & $\begin{array}{l}257.2 \\
{[245.6]}\end{array}$ & 234.1 & $\begin{array}{l}130.1 \\
{[270.7]}\end{array}$ & 411.2 \\
\hline & Deformability modulus for $0.5 \mathrm{f}_{\mathrm{ct}-\mathrm{DT}}, \mathrm{E}_{\mathrm{u} 50}(\mathrm{MPa})$ & & & & & $\begin{array}{l}1212.7 \\
{[985.2]}\end{array}$ & 757.6 & $\begin{array}{l}267.7 \\
{[907.6]}\end{array}$ & 1538.5 \\
\hline & Unit weight, $\gamma\left(\mathrm{kN} / \mathrm{m}^{3}\right)$ & & & & & $(* *)$ & $(* *)$ & $\begin{array}{l}15.11 \\
{[15.15]}\end{array}$ & 15.18 \\
\hline & Final water content, $\mathrm{w}_{\mathrm{f}}(\%)$ & & & & & $\begin{array}{l}68.8 \\
{[68.1]}\end{array}$ & 67.3 & $\begin{array}{l}60.0 \\
{[60.0]}\end{array}$ & 60.1 \\
\hline \multirow[t]{4}{*}{ FS test } & Flexural strength, $\mathrm{f}_{\mathrm{cf}-\mathrm{F}}(\mathrm{kPa})$ & $(*)$ & & $(*)$ & & $\begin{array}{l}1983.7 \\
{[1914.8]}\end{array}$ & 1845.9 & $\begin{array}{l}2277.6 \\
{[2194.9]}\end{array}$ & 2112.2 \\
\hline & Deflection, $\delta_{\mathrm{v}}(\mathrm{mm})$ & & & & & $\begin{array}{l}0.46 \\
{[0.46]}\end{array}$ & 0.46 & $\begin{array}{l}1.60 \\
{[1.63]}\end{array}$ & 1.66 \\
\hline & Unit weight, $\gamma\left(\mathrm{kN} / \mathrm{m}^{3}\right)$ & & & & & $\begin{array}{l}14.90 \\
{[14.89]}\end{array}$ & 14.87 & $\begin{array}{l}15.09 \\
{[15.15]}\end{array}$ & 15.20 \\
\hline & Final water content, $\mathrm{w}_{\mathrm{f}}(\%)$ & & & & & $\begin{array}{l}65.6 \\
{[65.2]}\end{array}$ & 64.8 & $\begin{array}{l}53.8 \\
{[54.3]}\end{array}$ & 54.8 \\
\hline
\end{tabular}

$[\ldots]-$ Average value; $\left({ }^{*}\right)$ Test not carried out; $\left({ }^{* *}\right)$ Data lost; $\mathrm{T}_{\mathrm{i}}-$ Test $\mathrm{i}$.

( $a_{w}$, ratio of the dry weight of binder to the dry weight of the soil) induces the increases of the compressive (UCS tests) and tensile strength (STS and FS tests) due to the increment in the cement reactions, creating stronger cementation bonds between the soil particles. These data are corroborated by the decreases in the final water content (Tables 2 and 3 ) due to the water consumed in the cement reactions. This beneficial impact of the BQ on the strength has also been reported by several other researchers, for example Kaniraj and Havanagi (2001), Kumar et al. (2007), Correia (2011) and Olgun (2013).

The results from the UCS (Fig. 1a), STS (Fig. 1b) and FS tests (Fig. 1d) are consistent with each other and illustrate the significant impact that the addition of polypropylene fibres to the soilbinder paste has, in terms of the stress-strain (UCS test) or load-displacement (STS and FS tests) behaviour, namely: (i) in general the tests show the decreasing of the stiffness with the addition of fibres, due to the inclusion of a more deformable material (fibres) into the soil-binder matrix; (ii) in general, the inclusion of fibres tends to decrease the peak compressive strength, which is explained by two cumulative effects: the presence of the fibres, which does not allow the creation of some cementation bonds between the soil particles; while the high binder content induces a very stiff composite material, with low deformations at peak, which are not enough to mobilize the tensile strength of the polypropylene fibres (extensible fibres), and consequently, these do not contribute to the increment of the strength; (iii) the inclusion of fibres in the stabilised soil changes its behaviour, from a brittle behaviour, with a significant reduction of strength after peak for the non-reinforced material, to a ductile behaviour without apparent loss of strength after peak, which is a consequence of the mobilization of the tensile strength of the fibres for higher deformations, as illustrated in Fig. 1. Although the effect of the fibres, in terms of ductility improvement, is corroborated by the generality of the works published (Consoli et al., 2009; Olgun, 2013), the detrimental effect of the inclusion of the fibres in terms of compressive strength, is only reported in a few works. Consoli et al. (2009) obtained the same trend for the compressive strength by using a high binder content in a sandy soil, while Khattak and Alrashidi (2006) observed a reduction of tensile strength (STS tests) for high plasticity clays stabilized with $10 \%$ of cement and reinforced with fibre contents higher than $0.3 \%$. As the stabilization of the soft soil under study requires a high binder content to achieve a significant level of improvement, the effect of the fibres is detrimental in terms of peak compressive strength.

The load-displacement behaviour from the DTS tests (Fig. 1c) does not match that observed in the remaining tests, since the stiffness is not affected by the presence of fibres and it continues to be brittle, although the loss of strength after peak is lower with the inclusion of fibres. This behaviour is explained by the fact that the reduced deformation at peak (less than $0.03 \%$ ) does not allow the tensile strength of the fibres, thus the stiffness is almost unaltered. After failure, the residual strength observed is only due to the mobilization of the tensile strength of the fibres and as the fibre content in both specimens is constant, the residual strength is naturally the same. 
Table 4

Effect of the fibre quantity - soil stabilised with a constant binder quantity of $375 \mathrm{~kg} / \mathrm{m}^{3}$ (Custódio, 2013).

\begin{tabular}{|c|c|c|c|c|c|c|c|c|c|c|c|}
\hline \multirow[t]{3}{*}{ Type of test } & \multirow[t]{3}{*}{ Property } & \multicolumn{10}{|c|}{ Fibre quantity $\left(\mathrm{kg} / \mathrm{m}^{3}\right)$} \\
\hline & & \multicolumn{2}{|l|}{0} & \multicolumn{2}{|l|}{25} & \multicolumn{2}{|l|}{50} & \multicolumn{2}{|l|}{75} & \multicolumn{2}{|l|}{100} \\
\hline & & $\mathrm{T}_{1}$ & $\mathrm{~T}_{2}$ & $\mathrm{~T}_{1}$ & $\mathrm{~T}_{2}$ & $\mathrm{~T}_{1}$ & $\mathrm{~T}_{2}$ & $\mathrm{~T}_{1}$ & $\mathrm{~T}_{2}$ & $\mathrm{~T}_{1}$ & $\mathrm{~T}_{2}$ \\
\hline & Binder quantity, $\mathrm{BQ}\left(\mathrm{kg} / \mathrm{m}^{3}\right)$ & 375 & & 375 & & 375 & & 375 & & 375 & \\
\hline & Binder content, $\mathrm{a}_{\mathrm{w}}(\%)$ & 53.6 & & 53.6 & & 53.6 & & 53.6 & & 53.6 & \\
\hline \multirow[t]{4}{*}{ UCS test } & Unconfined compressive strength, $\mathrm{q}_{\mathrm{u}}(\mathrm{kPa})$ & $\begin{array}{l}2761.0 \\
{[2623.4]}\end{array}$ & 2485.9 & $\begin{array}{l}2115.5 \\
{[2105.5]}\end{array}$ & 2095.4 & $\begin{array}{l}2173.0 \\
{[2111.0]}\end{array}$ & 2049.0 & $\begin{array}{l}2482.0 \\
{[2416.3]}\end{array}$ & 2350.6 & $\begin{array}{l}3167.2 \\
{[2939.2]}\end{array}$ & 2711.1 \\
\hline & Deformability modulus for $0.5 \mathrm{q}_{\mathrm{u}}, \mathrm{E}_{\mathrm{u} 50}(\mathrm{MPa})$ & $\begin{array}{l}193.5 \\
{[190.3]}\end{array}$ & 187.1 & $\begin{array}{l}155.4 \\
{[172.7]}\end{array}$ & 190.1 & $\begin{array}{l}231.0 \\
{[212.4]}\end{array}$ & 193.7 & $\begin{array}{l}157.6 \\
{[186.5]}\end{array}$ & 215.4 & $\begin{array}{l}236.6 \\
{[215.1]}\end{array}$ & 193.6 \\
\hline & Unit weight, $\gamma\left(\mathrm{kN} / \mathrm{m}^{3}\right)$ & $\begin{array}{l}15.43 \\
{[15.46]}\end{array}$ & 15.49 & $\begin{array}{l}15.76 \\
{[15.82]}\end{array}$ & 15.88 & $\begin{array}{l}15.45 \\
{[15.44]}\end{array}$ & 15.43 & $\begin{array}{l}15.27 \\
{[15.30]}\end{array}$ & 15.33 & $\begin{array}{l}14.75 \\
{[14.73]}\end{array}$ & 14.70 \\
\hline & Final water content, $\mathrm{w}_{\mathrm{f}}(\%)$ & $\begin{array}{l}68.7 \\
{[68.7]}\end{array}$ & 68.7 & $\begin{array}{l}70.8 \\
{[71.0]}\end{array}$ & 71.3 & $\begin{array}{l}70.0 \\
{[70.5]}\end{array}$ & 71.1 & $\begin{array}{l}70.3 \\
{[72.0]}\end{array}$ & 73.6 & $\begin{array}{l}71.7 \\
{[71.9]}\end{array}$ & 72.0 \\
\hline \multirow[t]{4}{*}{ STS test } & Split tensile strength, $\mathrm{f}_{\mathrm{ct}-\mathrm{ST}}(\mathrm{kPa})$ & $\begin{array}{l}342.2 \\
{[340.4]}\end{array}$ & 338.5 & $\begin{array}{l}408.8 \\
{[423.9]}\end{array}$ & 439.0 & $\begin{array}{l}454.8 \\
{[454.8]}\end{array}$ & 454.8 & $\begin{array}{l}618.8 \\
{[590.0]}\end{array}$ & 561.1 & $\begin{array}{l}607.0 \\
{[578.9]}\end{array}$ & 550.8 \\
\hline & Vertical displacement at failure, $\delta_{\mathrm{v}}(\mathrm{mm})$ & $\begin{array}{l}1.12 \\
{[1.17]}\end{array}$ & 1.22 & $\begin{array}{l}3.85 \\
{[4.09]}\end{array}$ & 4.32 & $\begin{array}{l}3.81 \\
{[4.88]}\end{array}$ & 5.95 & $\begin{array}{l}12.50 \\
{[7.70]}\end{array}$ & 2.90 & $\begin{array}{l}12.32 \\
{[12.41]}\end{array}$ & 12.50 \\
\hline & Unit weight, $\gamma\left(\mathrm{kN} / \mathrm{m}^{3}\right)$ & $\begin{array}{l}15.32 \\
{[15.27]}\end{array}$ & 15.22 & $\begin{array}{l}15.07 \\
{[15.27]}\end{array}$ & 15.46 & $\begin{array}{l}15.19 \\
{[15.43]}\end{array}$ & 15.67 & $\begin{array}{l}15.77 \\
{[15.72]}\end{array}$ & 15.67 & $\begin{array}{l}15.41 \\
{[15.16]}\end{array}$ & 14.90 \\
\hline & Final water content, $\mathrm{w}_{\mathrm{f}}(\%)$ & $\begin{array}{l}67.2 \\
{[67.1]}\end{array}$ & 67.0 & $\begin{array}{l}65.5 \\
{[66.3]}\end{array}$ & 67.0 & $\begin{array}{l}68.1 \\
{[68.2]}\end{array}$ & 68.4 & $\begin{array}{l}65.5 \\
{[66.99]}\end{array}$ & 68.4 & $\begin{array}{l}64.4 \\
{[65.2]}\end{array}$ & 66.0 \\
\hline \multirow[t]{4}{*}{ DTS test } & Direct tensile strength, $\mathrm{f}_{\mathrm{ct}-\mathrm{DT}}(\mathrm{kPa})$ & $\begin{array}{l}411.2 \\
{[378.6]}\end{array}$ & 345.9 & $\begin{array}{l}171.8 \\
{[195.2]}\end{array}$ & 218.6 & $\begin{array}{l}257.2 \\
{[245.7]}\end{array}$ & 234.2 & $\begin{array}{l}218.8 \\
{[152.6]}\end{array}$ & 86.4 & $\begin{array}{l}163.8 \\
{[167.7]}\end{array}$ & 171.7 \\
\hline & Deformability modulus for $0.5 \mathrm{f}_{\mathrm{ct}-\mathrm{DT}}, \mathrm{E}_{\mathrm{u} 50}(\mathrm{MPa})$ & $\begin{array}{l}1413.6 \\
{[1534.4]}\end{array}$ & 1655.3 & $\begin{array}{l}524.7 \\
{[433.7]}\end{array}$ & 342.8 & $\begin{array}{l}1212.7 \\
{[985.2]}\end{array}$ & 757.6 & $\begin{array}{l}364.7 \\
{[229.2]}\end{array}$ & 93.6 & $\begin{array}{l}131.1 \\
{[141.0]}\end{array}$ & 150.9 \\
\hline & Unit weight, $\gamma\left(\mathrm{kN} / \mathrm{m}^{3}\right)$ & $\begin{array}{l}15.45 \\
{[14.44]}\end{array}$ & 15.43 & $\begin{array}{l}14.78 \\
{[14.65]}\end{array}$ & 14.51 & $(* *)$ & $(* *)$ & $(* *)$ & $(* *)$ & $\begin{array}{l}14.78 \\
{[14.65]}\end{array}$ & 14.51 \\
\hline & Final water content, $\mathrm{w}_{\mathrm{f}}(\%)$ & $\begin{array}{l}70.7 \\
{[70.4]}\end{array}$ & 70.0 & $\begin{array}{l}67.1 \\
{[67.4]}\end{array}$ & 67.6 & $\begin{array}{l}68.8 \\
{[68.1]}\end{array}$ & 67.3 & $\begin{array}{l}69.9 \\
{[69.4]}\end{array}$ & 68.9 & $\begin{array}{l}70.4 \\
{[69.4]}\end{array}$ & 68.4 \\
\hline \multirow[t]{4}{*}{ FS test } & Flexural strength, $\mathrm{f}_{\mathrm{cf}-\mathrm{F}}(\mathrm{kPa})$ & $\begin{array}{l}760.5 \\
{[774.0]}\end{array}$ & 787.5 & $\begin{array}{l}1515.3 \\
{[1432.7]}\end{array}$ & 1350.0 & $\begin{array}{l}1983.7 \\
{[1914.8]}\end{array}$ & 1845.9 & $\begin{array}{l}1735.7 \\
{[1924.0]}\end{array}$ & 2112.2 & $\begin{array}{l}2084.7 \\
{[1855.1]}\end{array}$ & 1625.5 \\
\hline & Deflection, $\delta_{\mathrm{v}}(\mathrm{mm})$ & $\begin{array}{l}0.09 \\
{[0.10]}\end{array}$ & 0.11 & $\begin{array}{l}1.05 \\
{[1.08]}\end{array}$ & 1.10 & $\begin{array}{l}0.46 \\
{[0.46]}\end{array}$ & 0.46 & $\begin{array}{l}1.08 \\
{[1.59]}\end{array}$ & 2.09 & $\begin{array}{l}1.98 \\
{[1.45]}\end{array}$ & 0.92 \\
\hline & Unit weight, $\gamma\left(\mathrm{kN} / \mathrm{m}^{3}\right)$ & $\begin{array}{l}15.11 \\
{[15.04]}\end{array}$ & 14.97 & $\begin{array}{l}(* *) \\
{[15.30]}\end{array}$ & 15.30 & $\begin{array}{l}14.90 \\
{[14.89]}\end{array}$ & 14.87 & $\begin{array}{l}15.11 \\
{[15.09]}\end{array}$ & 15.06 & $\begin{array}{l}14.99 \\
{[14.97]}\end{array}$ & 14.94 \\
\hline & Final water content, $\mathrm{w}_{\mathrm{f}}(\%)$ & $\begin{array}{l}66.2 \\
{[66.1]}\end{array}$ & 66.0 & $\begin{array}{l}63.7 \\
{[66.2]}\end{array}$ & 68.7 & $\begin{array}{l}65.6 \\
{[65.2]}\end{array}$ & 64.8 & $\begin{array}{l}64.3 \\
{[64.7]}\end{array}$ & 65.1 & $\begin{array}{l}62.4 \\
{[63.9]}\end{array}$ & 65.5 \\
\hline
\end{tabular}

$[\ldots]$ - Average value; $\left({ }^{* *}\right)$ Data lost; $\mathrm{T}_{\mathrm{i}}-$ Test $\mathrm{I}$.

Fig. 2 depicts the evolution, with $\mathrm{BQ}$ of the compressive strength $\left(q_{u}\right)$, the tensile strength (STS: $\mathrm{f}_{\mathrm{ct}-\mathrm{STS}}$; DTS: $\left.\mathrm{f}_{\mathrm{ct}-\mathrm{DTS}} ; \mathrm{FS}: \mathrm{f}_{\mathrm{ct}-\mathrm{FS}}\right)$ and the Young's modulus evaluated for $50 \%$ of the compressive or tensile strength $\left(E_{\mathrm{u} 50}\right)$ for the four types of tests carried out, which clarify some of the features previously described from the analysis of Fig. 1. The results of the UCS (Fig. 2a) and STS tests (Fig. 2b) without fibres reveal a quasi-linear increase in the strength with BQ; the results of the DTS (Fig. 2c) and FS (Fig. 2d) tests also reveal an increment in the strength when BQ increases from 375 to $500 \mathrm{~kg} / \mathrm{m}^{3}$. The results also show that the inclusion of $50 \mathrm{~kg} / \mathrm{m}^{3}$ of fibres has a detrimental effect on $\mathrm{qu}_{\mathrm{u}}$ (Fig. 2a) and $\mathrm{f}_{\mathrm{ct}-\mathrm{DTS}}$ (Fig. 2c); this detrimental effect increases with the increment in $\mathrm{BQ}$ in relation to the case without fibres. In terms of stiffness, the increases of the BQ promote the increment in the $\mathrm{E}_{\mathrm{u} 50}$ (Fig. 2a, c), which is lower in the presence of fibres. The stiffness evaluated from the DTS tests (Fig. 2c) with the inclusion of fibres is almost unchanged despite the high scattering of the data.

The evolution of the strength ratio $\left(R_{\mathrm{qu}}=\mathrm{qu}[\mathrm{FQ} \neq 0] / \mathrm{q}_{\mathrm{u}}[\mathrm{FQ}=0]\right.$; $\left.R_{\mathrm{fct}}=\mathrm{f}_{\mathrm{ct}[\mathrm{FQ} \neq 0]} / \mathrm{f}_{\mathrm{ct}[\mathrm{FQ}=0]}\right)$ and the stiffness ratio $\left(R_{\mathrm{Eu}}=\mathrm{E}_{\mathrm{u} 50[\mathrm{FQ} \neq 0]} / \mathrm{E}_{\mathrm{u} 50}\right.$ $[\mathrm{FQ}=0]$ ) with $\mathrm{BQ}$ is illustrated in Fig. 3. In general, the strength ratio (Fig. 3a) varies between 2.5 (FS test) and 0.5 (DTS test), and tends to decrease with the increment of $\mathrm{BQ}$, that is, the addition of fibres has a beneficial impact on the peak compressive strength for a BQ of lower than $250 \mathrm{~kg} / \mathrm{m} 3$, while for a higher BQ the impact of the presence of fibres is slightly detrimental (UCS and DTS tests). The stiffness ratio (Fig. 3b) evaluated from the UCS tests varies between 0.60 and 1.12 , with a significant scattering of data, which does not show a clear tendency, i.e., this ratio has a maximum for a BQ of $375 \mathrm{~kg} / \mathrm{m}^{3}$, decreasing for higher and lower values of BQ. Additionally, the average $\mathrm{R}_{\mathrm{Eu}}$ evaluated from the DTS tests is lower than that obtained from the UCS tests. However, the comparison between the stiffness of the UCS and DTS tests should only be done in qualitative terms because the strain level used to evaluate the stiffness for both tests is completely different, as observed in Fig. 1.

Fig. 4 depicts the effect of the BQ on the variation of some relationships between the compressive and tensile strength $\left(\mathrm{f}_{\mathrm{ct}-\mathrm{DTS}} /\right.$ $\left.\mathrm{q}_{\mathrm{u}}, \mathrm{f}_{\mathrm{ct}-\mathrm{DTS}} / \mathrm{f}_{\mathrm{ct}-\mathrm{STS}}, \mathrm{f}_{\mathrm{ct}-\mathrm{DTS}} / \mathrm{f}_{\mathrm{ct}-\mathrm{FS}}, \mathrm{f}_{\mathrm{ct}-\mathrm{STS}} / \mathrm{q}_{\mathrm{u}}\right)$, which could be helpful at a pre-design stage. In general, Fig. 4a shows the decrease of the $\mathrm{f}_{\mathrm{ct}}$ DTS $/ \mathrm{q}_{\mathrm{u}}, \mathrm{f}_{\mathrm{ct}-\mathrm{DTS}} / \mathrm{f}_{\mathrm{ct}-\mathrm{STS}}$ and $\mathrm{f}_{\mathrm{ct}-\mathrm{DTS}} / \mathrm{f}_{\mathrm{ct}-\mathrm{FS}}$ with $\mathrm{BQ}$, with a more significant reduction for $\mathrm{f}_{\mathrm{ct}-\mathrm{DTS}} / \mathrm{f}_{\mathrm{ct}-\mathrm{STS}}$, mainly with the addition of $50 \mathrm{~kg} / \mathrm{m}^{3}$ of fibres. The ratio $\mathrm{f}_{\mathrm{ct}-\mathrm{DTS}} / \mathrm{f}_{\mathrm{ct}-\mathrm{STS}}$ varies between 0.94 and 1.11 without fibres and between 0.42 and 0.61 for an FQ of $50 \mathrm{~kg} / \mathrm{m}^{3}$; on the other hand $\mathrm{f}_{\mathrm{ct}-\mathrm{DTS}} / \mathrm{f}_{\mathrm{ct}-\mathrm{FS}}$ changes between 0.42 and 0.49 for tests without fibres, but becomes almost constant $\left(\mathrm{f}_{\mathrm{ct}-\mathrm{DTS}} / \mathrm{f}_{\mathrm{ct}-\mathrm{FS}} \cong 0.11\right.$ ) when $50 \mathrm{~kg} / \mathrm{m}^{3}$ of fibres is added to the mixture. It should be pointed out that these relationships are very different from those reported for concrete $\left(\mathrm{f}_{\mathrm{ct}-\mathrm{DTS}} / \mathrm{f}_{\mathrm{ct}-\mathrm{STS}}=0.9 ; \mathrm{f}_{\mathrm{ct}-\mathrm{DTS}} / \mathrm{f}_{\mathrm{ct}-\mathrm{FS}}=0.65\right)(\mathrm{NP}$ EN 1992-1-1, 2010). Fig. 4b shows that $\mathrm{f}_{\mathrm{ct}-\mathrm{STS}} / \mathrm{q}_{\mathrm{u}}$ changes to between 0.13 and 0.22 without fibres, but changes to $0.19-0.28$ with the inclusion of $50 \mathrm{~kg} / \mathrm{m}^{3}$ of fibres. These values are slightly higher than those obtained by Consoli et al. (2013c) with a stabilised sandy soil reinforced with fibres, $\mathrm{f}_{\mathrm{ct}-\mathrm{STS}} / q_{\mathrm{u}}=0.14$, which is explained by the different natures of the soils and the lower binder content used by Consoli et al. (2013c). 

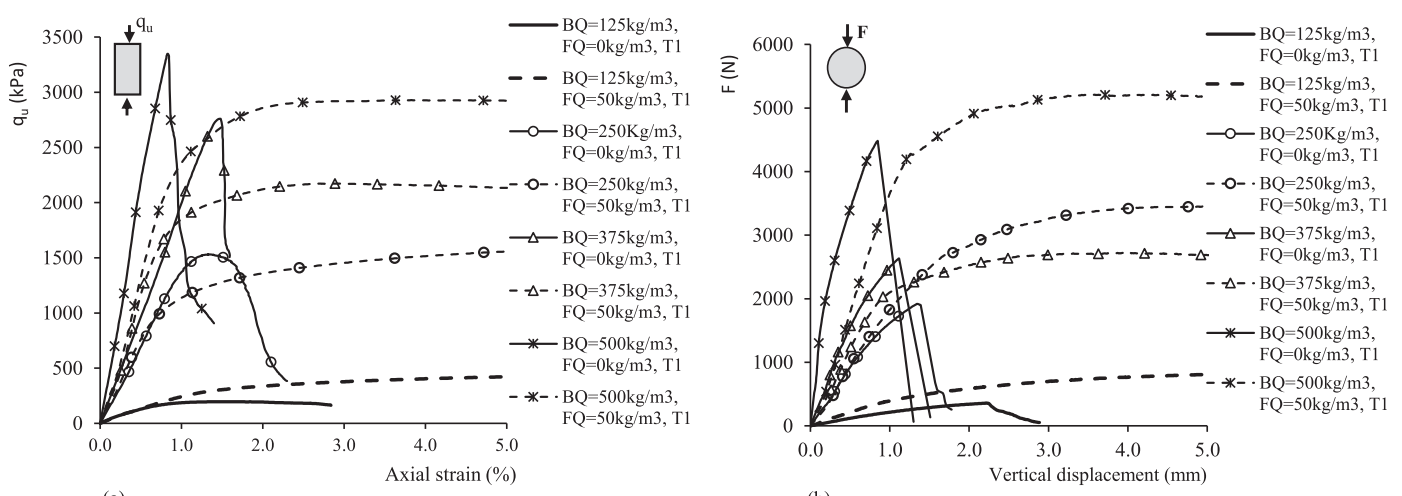

(a)

(b)
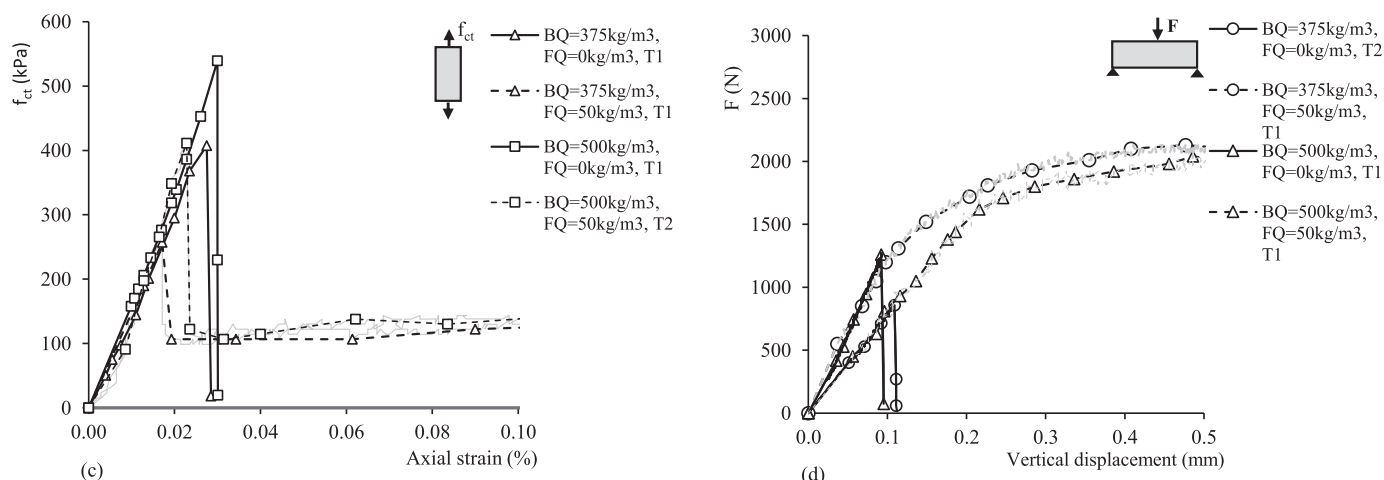

Fig. 1. Stress-strain or load-displacement curves. Effect of binder quantity, both with and without fibres $\left(F Q=50 \mathrm{~kg} / \mathrm{m}^{3}\right)$ : (a) UCS test; (b) STS test; (c) DTS test; (d) FS test.

\subsection{Effect of fibre quantity}

Fig. 5 and Table 4 show the results of the UCS, STS, DTS and FS tests, which illustrate the effect of the fibre quantity on the stress-

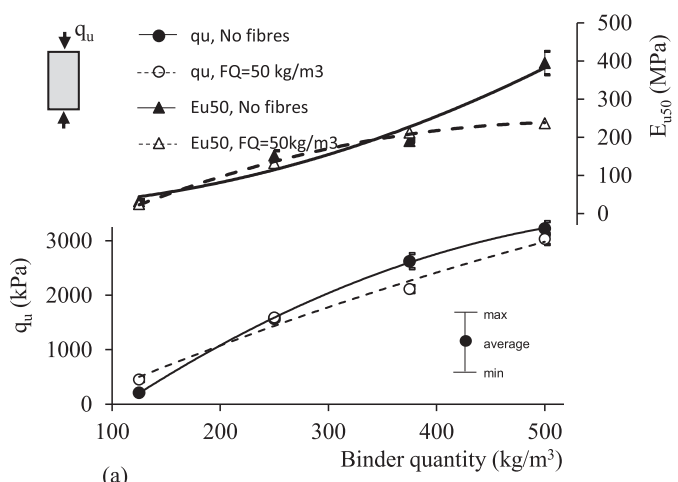

(a)
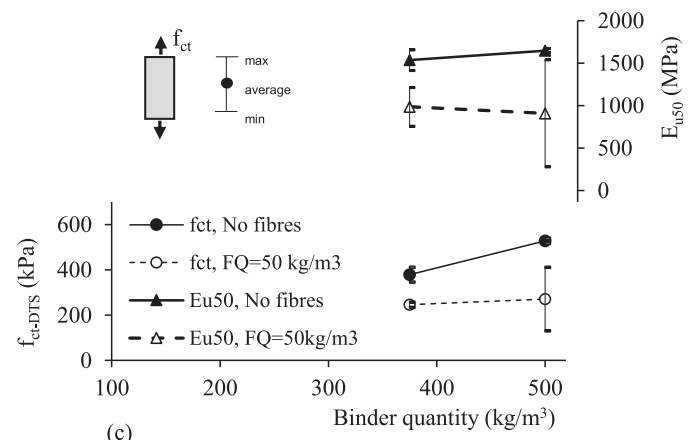

(c) strain or load-displacement behaviour. In general, the unit weight shows a tendency to decrease slightly with the increment of the fibres, due to the addition of a lighter material (fibres) to the soilbinder matrix. The results of the UCS (Fig. 5a), STS (Fig. 5b) and

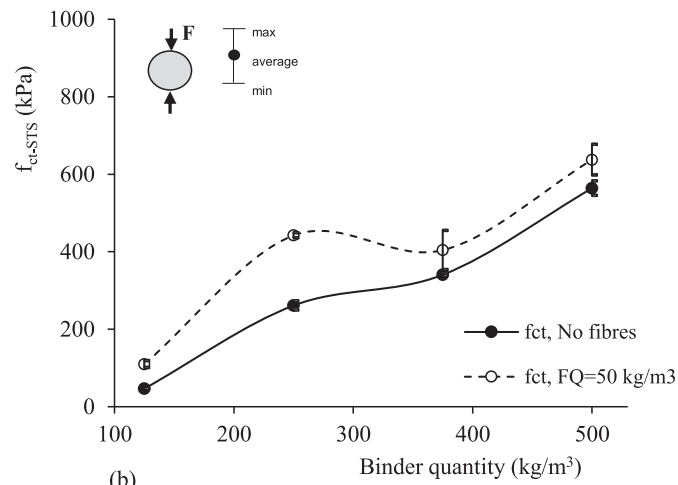

(b)

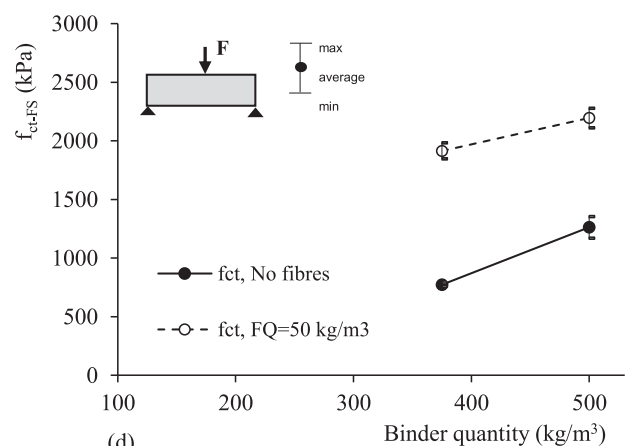

(d)

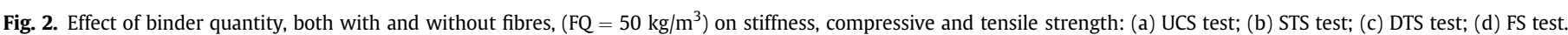




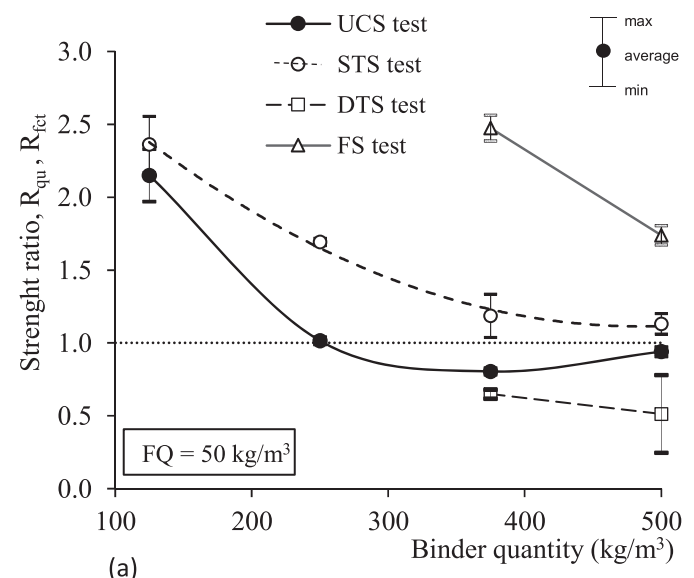

(a)

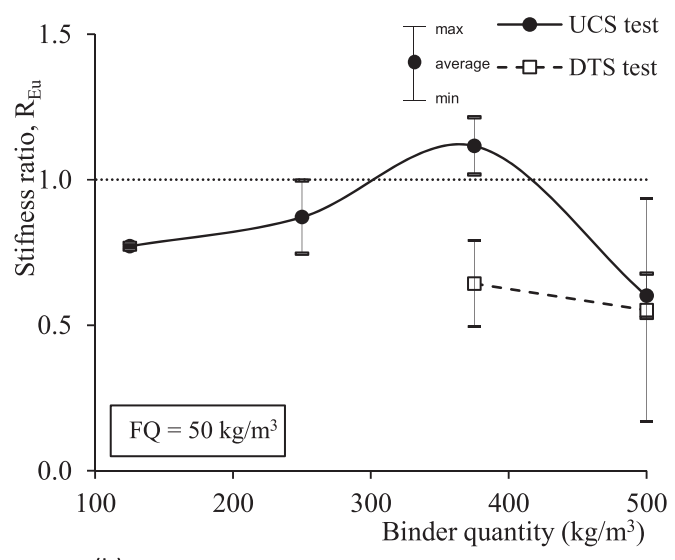

(b)

Fig. 3. Effect of binder quantity on: (a) strength ratio, $R_{q u}$; (b) stiffness ratio, $R_{E u}$ -

FS (Fig. 5d) tests indicate that the inclusion of an FQ of $25 \mathrm{~kg} / \mathrm{m}^{3}$ is sufficient to change the behaviour of the composite material, from a brittle (without fibres) to a ductile behaviour, which is consistent with the findings of Kaniraj and Havanaji (2001) and Olgun (2013). This behaviour is not reproduced in the DTS tests; indeed all the results (with and without fibres) indicate a brittle behaviour, although the inclusion of fibres decreases the loss of strength after peak, owing to the mobilization of the tensile strength of the fibres after the generalised failure of the cementitious matrix, which occurs for axial strains of $0.02 \%-0.03 \%$.

Fig. 6 summarizes the effect of FQ in terms of the stiffness, compressive and tensile strength, obtained from the four types of tests. The results reveal some common features, namely: (i) a decrease in the stiffness, the $q_{u}$ and the $\mathrm{f}_{\mathrm{ct}-\mathrm{DTs}}$ with the inclusion of $25 \mathrm{~kg} / \mathrm{m}^{3}$ of fibres in relation to the unreinforced material, because the presence of the fibres in the mixture prevents the formation of some cementation bonds with the soil particles; (ii) there is also a slight recovery of the strength and the stiffness with the increase in FQ inducing a peak of stiffness and strength when the amount of fibres is between 50 and $75 \mathrm{~kg} / \mathrm{m}^{3}$; the exception to this is shown by the results of the UCS test, which indicate a slight continuous recovery of $q_{u}$ with the increment of FQ; (iii) with the exception of the results of the DTS tests and the results of the UCS for an FQ lower than $100 \mathrm{~kg} / \mathrm{m}^{3}$, the addition of fibres to the soil-binder mixture increases the strength in relation to the non-reinforced material. In fact, for any of the two tensile tests (STS and FS) the tensile strength increases with the increment of fibre content, reaches a maximum value for an optimum fibre quantity of $50-75 \mathrm{~kg} / \mathrm{m}^{3}$ and after that starts to decrease; this behaviour is in line with the findings of Khattak and Alrashidi (2006) for STS tests.

In general, the results of UCS and DTS tests seem to indicate that, for higher binder contents, the behaviour of the composite material is fundamentally governed by the cementitious bonds which are responsible for the high strength and stiffness of the composite material, therefore the tensile strength of the polypropylene fibres (more extensible) is not mobilized before the peak strength that occurs for very low deformations. Accordingly, in UCS and DTS tests the addition of fibres does not contribute to improving the mechanical behaviour of the composite material for such binder contents.

Fig. 7 depicts the effect of the fibre quantity on the strength and the stiffness ratios, which, in general, reveals a non-linear evolution and a significant reduction of the $\mathrm{R}_{\mathrm{qu}}, \mathrm{R}_{\mathrm{fct}}$ (DTS tests) and $\mathrm{R}_{\mathrm{Eu}}$ with the inclusion of $25 \mathrm{~kg} / \mathrm{m}^{3}$ of fibres, reflecting the abovementioned behaviour. The FS tests lead to a higher $\mathrm{R}_{\mathrm{fct}}(1.85-2.49)$, while the lowest results are induced by the DTS test, with an $\mathrm{R}_{\mathrm{fct}}$ of $0.40-0.64$. Despite the high level of scattering, $\mathrm{R}_{\mathrm{Eu}}$ also exhibits distinct tendencies depending on the type of test (UCS or DTS); $R_{\mathrm{Eu}}$ evaluated by DTS tests decreases with FQ while the UCS tests induce an almost constant stiffness ratio. Thus, the results indicate that the efficacy of the fibre reinforcement seems to be dependent on the strain level induced by each test at failure, linked with the higher or lower mobilization of the tensile strength of the fibres, which is related with the binder content. In this way, the FS test originates a

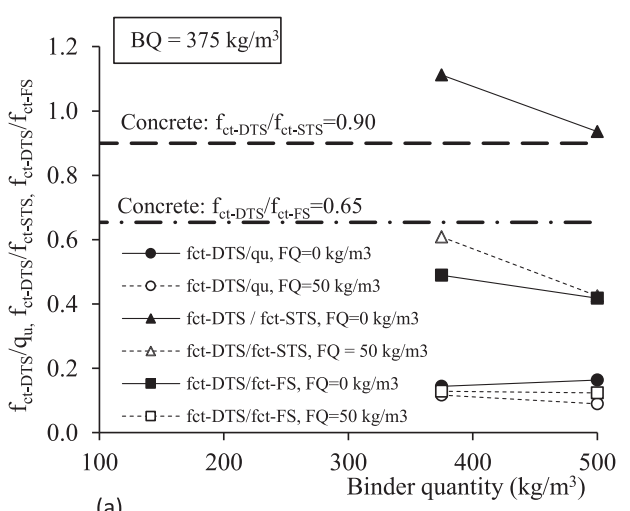

(a)

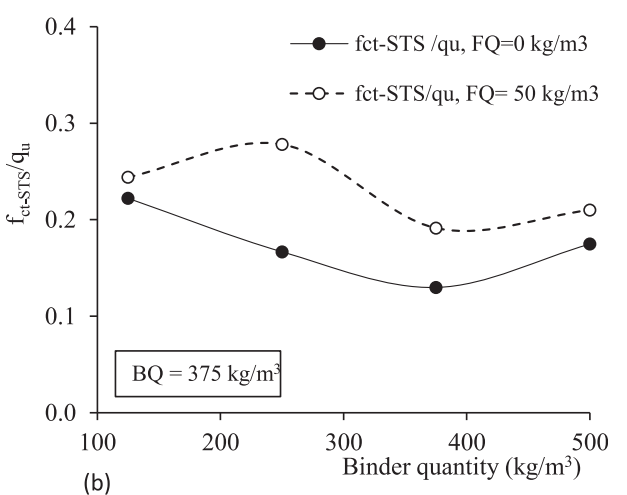

(b)

Fig. 4. Effect of binder quantity on the ratio of peak strength: (a) $f_{c t-D T S} / q_{u}, f_{c t-D T S} / f_{c t-S T S}, f_{c t-D T S} / f_{c t-F s} ;(b) f_{c t-S T S} / q_{u}$ - 

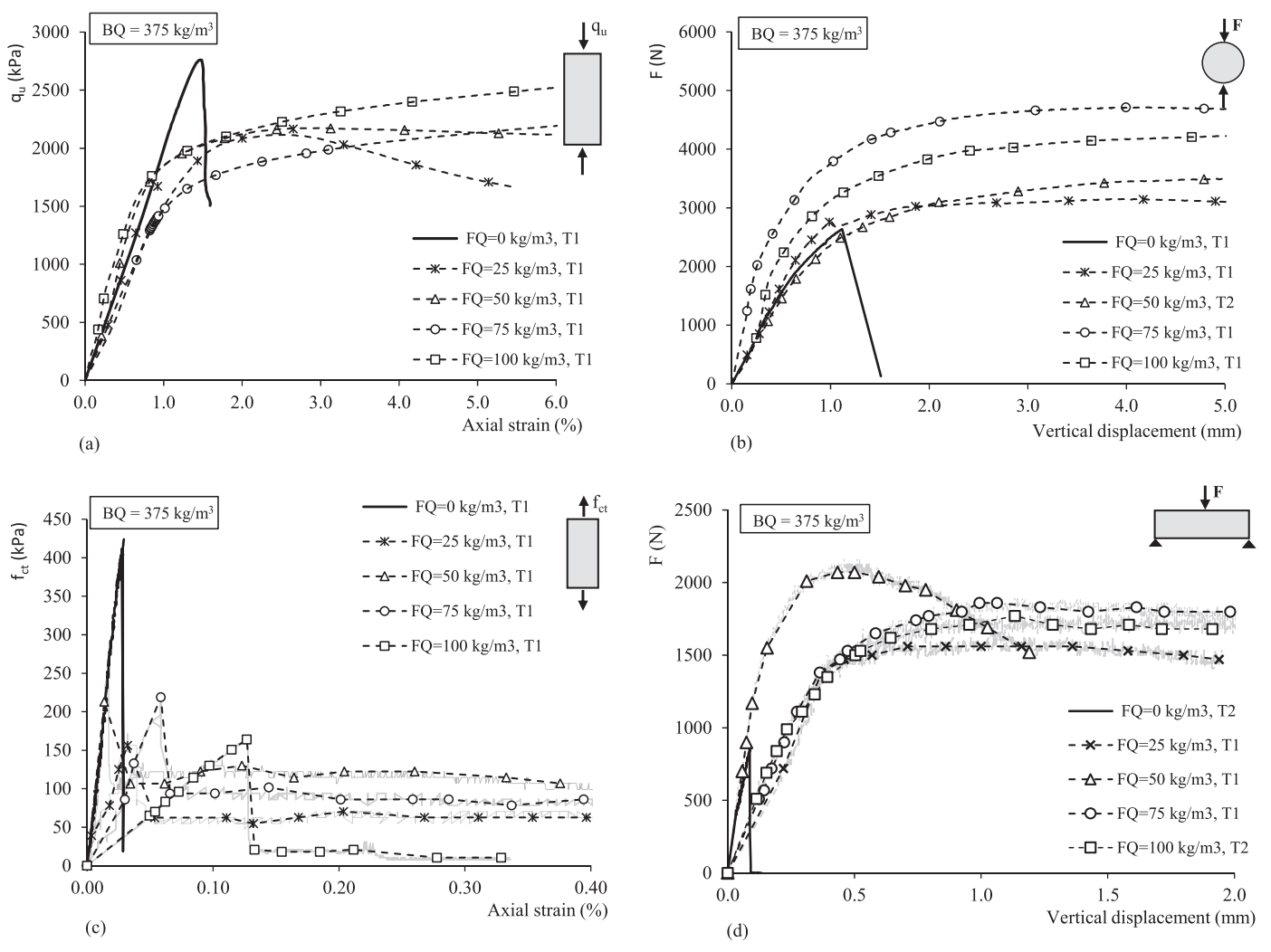

Fig. 5. Stress-strain or load-displacement curves. Effect of fibre quantity: (a) UCS test; (b) STS test; (c) DTS test; (d) FS test.
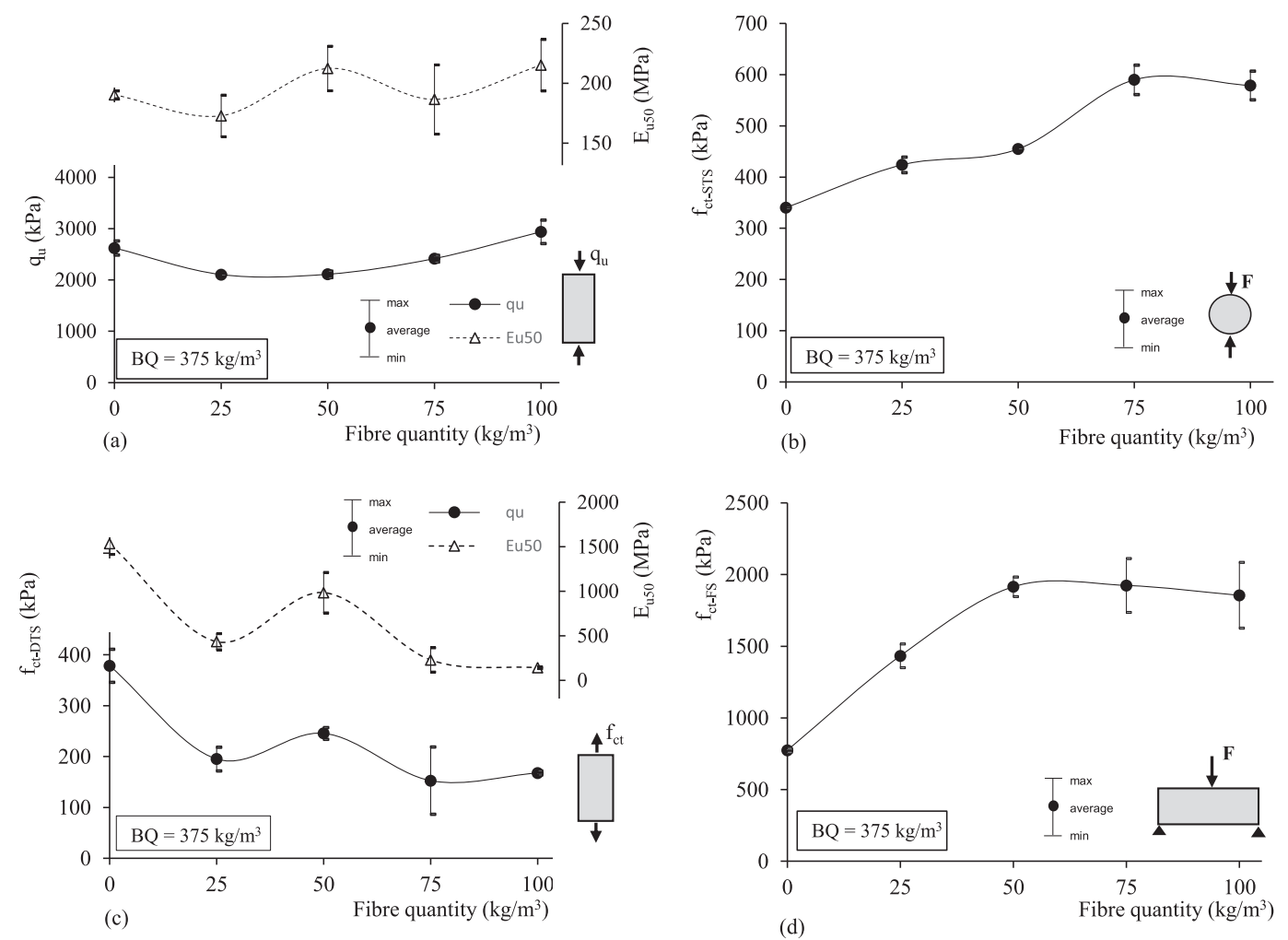

Fig. 6. Effect of fibre quantity on stiffness, compressive and tensile strength. (a) UCS test; (b) STS test; (c) DTS test; (d) FS test. 


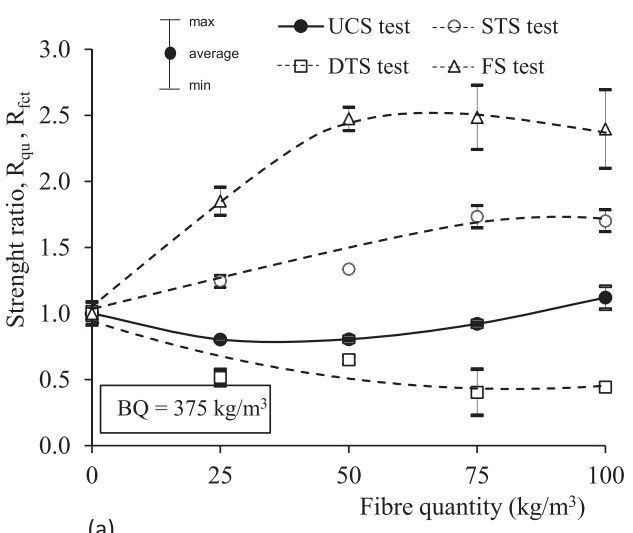

(a)

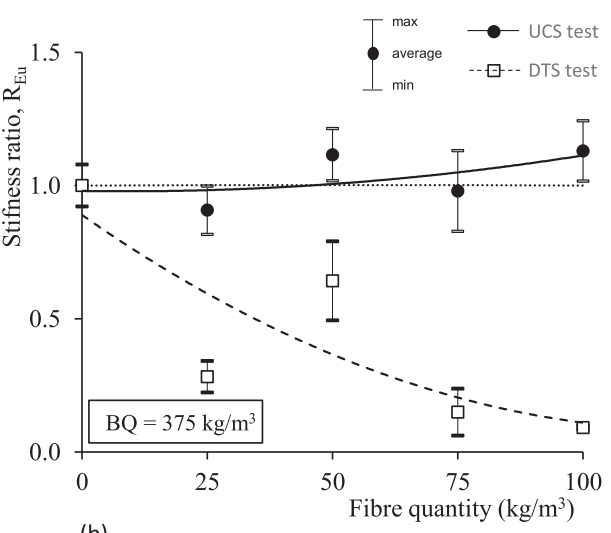

(b)

Fig. 7. Effect of fibre quantity on: (a) strength ratio, $\mathrm{R}_{\mathrm{qu}}$; (b) stiffness ratio, $\mathrm{R}_{\mathrm{Eu}}$.

more deformable mechanism than the other three tests, thus the fibres play a more relevant role. The DTS test contrast directly with this since the failure occurs for very low deformations, which are not enough to mobilize the tensile strength of the fibres and their presence in the paste impedes the creation of some cementation bonds.

The effect of the FQ on the variation of some relationships between the compressive and tensile strength $\left(\mathrm{f}_{\mathrm{ct}-\mathrm{DTS}} / \mathrm{q}_{\mathrm{u}}, \mathrm{f}_{\mathrm{ct}-\mathrm{DTS}} / \mathrm{f}_{\mathrm{ct}-\mathrm{STS}}\right.$, $\left.\mathrm{f}_{\mathrm{ct}-\mathrm{DTS}} / \mathrm{f}_{\mathrm{ct}-\mathrm{FS}}, \mathrm{f}_{\mathrm{ct}-\mathrm{STS}} / \mathrm{q}_{\mathrm{u}}\right)$ is illustrated in Fig. 8. The relationship $\mathrm{f}_{\mathrm{ct}-\mathrm{DTS}} /$ $\mathrm{f}_{\mathrm{ct}-\mathrm{STS}}$ decreases greatly with the increment of FQ, while the remaining relationships exhibit a small change with $\mathrm{FQ}$ with the following variations: $\mathrm{f}_{\mathrm{ct}-\mathrm{DTS}} / q_{\mathrm{u}}=0.06-0.12 ; \mathrm{f}_{\mathrm{ct}-\mathrm{STS}} / q_{\mathrm{u}}=0.20-0.24$; $\mathrm{f}_{\mathrm{ct}-\mathrm{DTS}} / \mathrm{f}_{\mathrm{ct}-\mathrm{FS}}=0.09-0.14$. As seen previously, the ratio $\mathrm{f}_{\mathrm{ct}-\mathrm{STS}} / \mathrm{q}_{\mathrm{u}}$ is slightly higher than that obtained by Consoli et al. (2013c) for a cemented sandy soil reinforced with polypropylene fibres, $\left(\mathrm{f}_{\mathrm{ct}-\mathrm{STS}} /\right.$ $q_{\mathrm{u}}=0.14$ ) and by Kumar et al. (2007) for ash-soil mixtures stabilized with lime and reinforced with polypropylene fibres ( $\mathrm{f}_{\mathrm{ct}-\mathrm{STS}}$ ) $\mathrm{qu}=0.14-0.17$, increasing with the fibre content). As described previously in the analysis of the effect of binder quantity, the $\mathrm{f}_{\mathrm{ct}-\mathrm{DTS}} /$ $\mathrm{f}_{\mathrm{ct}-\mathrm{STS}}$ (with fibres) and $\mathrm{f}_{\mathrm{ct}-\mathrm{DTS}} / \mathrm{f}_{\mathrm{ct}-\mathrm{FS}}$ relationships are much lower from those stated for concrete (NP EN 1992-1-1, 2010), $\mathrm{f}_{\mathrm{ct}-\mathrm{DTS}} / \mathrm{f}_{\mathrm{ct}-}$ STS $=0.9$ and $\mathrm{f}_{\mathrm{ct}-\mathrm{DTS}} / \mathrm{f}_{\mathrm{ct}-\mathrm{FS}}=0.65$, which is due to a different matrix.

\section{Conclusions}

Regarding the results of the unconfined compressive strength (UCS) tests, split tensile strength (STS) tests, direct tensile strength (DTS) tests and flexural strength (FS) tests carried out with stabilised specimens of the "Baixo Mondego" soft soil, both reinforced

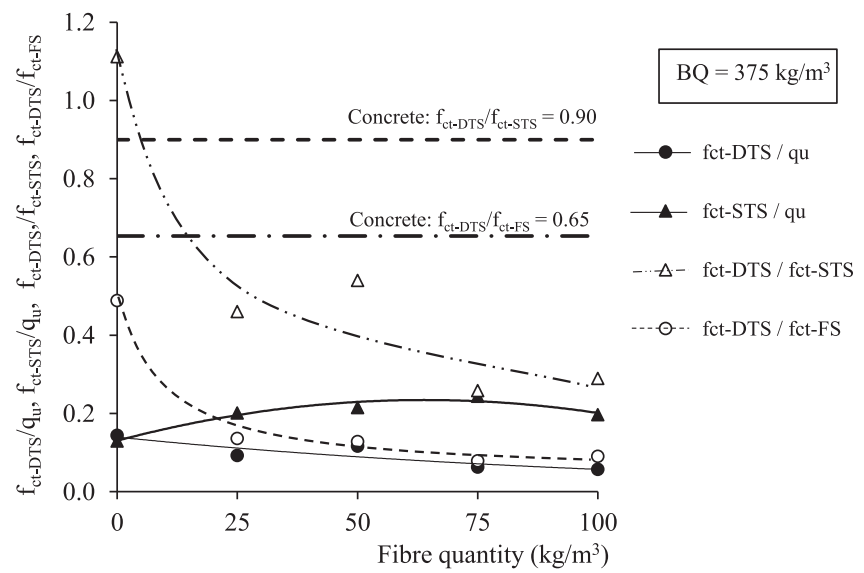

Fig. 8. Effect of fibre quantity on the ratio of peak strength: fct-DTS/qu, fct-STS/qu, fctDTS/fct-STS and fct-DTS/fct-FS. and non-reinforced with polypropylene fibres, the following observations and conclusions can be drawn:

(i) The increment of binder quantity improves the mechanical characteristics of the stabilised material (i.e., the stiffness, the compressive and the tensile strength increase), however the presence of fibres may reduce this beneficial effect (UCS and DTS tests).

(ii) In general, the effect of the fibre quantity on the stiffness, compressive and tensile strength exhibits a non-linear trend, i.e., the mechanical properties are not proportional to the amount of fibres added to the paste. In fact the inclusion of $25 \mathrm{~kg} / \mathrm{m}^{3}$ of polypropylene fibres into the soil-binder mixtures tends to decrease the peak strength, both under compression and tension (DTS tests), which is explained by the fact that the fibres do not allow some cementation bonds to be established with the soil particles. However, an addition of $50-75 \mathrm{~kg} / \mathrm{m}^{3}$ of fibres leads to an improvement in the mechanical characteristics, in relation to both lower and higher quantities of fibres, indicating that this is the optimum value.

(iii) With the exception of the DTS tests, the results of the other tests indicate that the inclusion of fibres into the soil-binder paste changes the behaviour of the material from brittle to more ductile. In fact, the main repercussion of the inclusion of fibres in the specimens submitted to DTS test is to reduce the loss of strength after peak, now exhibiting a nonnegligible residual strength, which corresponds to the mobilized tensile strength of the fibres.

(iv) The effect associated with the addition of fibres depends on the type of the tests, namely, the strain level imposed by each test at failure. Thus, the failure in the DTS test occurs for very low deformations, which are insufficient to mobilize the tensile strength of the fibres, so the impact of the fibres on the peak strength is negligible. On the other hand, the FS test imposes a more deformable mechanism, which allows the mobilization of the tensile strength of the fibres, thus the fibres have a more important effect on the strength.

(v) In general, the relationships $\mathrm{f}_{\mathrm{ct}-\mathrm{DTS}} / \mathrm{f}_{\mathrm{ct}-\mathrm{FS}}, \mathrm{f}_{\mathrm{ct}-\mathrm{DTS}} / \mathrm{f}_{\mathrm{ct}-\mathrm{STS}}, \mathrm{f}_{\mathrm{ct}-\mathrm{DTS}} /$ $\mathrm{q}_{\mathrm{ut}}$ and $\mathrm{f}_{\mathrm{ct}-\mathrm{STS}} / \mathrm{q}_{\mathrm{u}}$, show a decrease with the increment of the binder and fibre quantities.

\section{Acknowledgements}

The authors would like to express their thanks to CIMPOR and to Biu International/Bekaert for supplying, respectively, the binders and the fibres used in the work and to the institutions that 
financially supported the research: CIEC, CICC and FCT (PTDC/ECM/ 101875/2008).

\section{Notation}

The following symbols are used in this paper (basic SI units are given in parentheses):

$\mathrm{a}_{\mathrm{w}} \quad$ binder content (\%)[ratio of the dry weight of binder to the dry weight of the soil];

BQ binder quantity $\left(\mathrm{kg} / \mathrm{m}^{3}\right)$ [dry weight of binder per volume of soil];

DTS direct tensile strength;

$\mathrm{E}_{\mathrm{u} 50}$ secant Young's modulus for 0.5qu (Pa);

$\mathrm{F}$ force $(\mathrm{N})$;

$\mathrm{f}_{\mathrm{ct}} \quad$ maximum tensile strength $(\mathrm{Pa})$;

$\mathrm{f}_{\mathrm{ct}-\mathrm{DTS}}$ maximum tensile strength evaluated from DTS tests (Pa);

$\mathrm{f}_{\mathrm{ct}-\mathrm{FS}} \quad$ maximum tensile strength evaluated from FS tests ( $\left.\mathrm{Pa}\right)$;

$\mathrm{f}_{\mathrm{ct}-\mathrm{STS}} \quad$ maximum tensile strength evaluated from STS tests $(\mathrm{Pa})$;

FQ fiber quantity $\left(\mathrm{kg} / \mathrm{m}^{3}\right)$ [weight of fibers per volume of soil];

FS flexural strength;

$\mathrm{q}_{\mathrm{u}} \quad$ maximum unconfined compressive strength (Pa);

$\mathrm{R}_{\mathrm{qu}} \quad$ strength ratio $\left.\left(\mathrm{q}_{\mathrm{u}[\mathrm{FQ} \neq 0]} / \mathrm{qu}_{\mathrm{u}} \mathrm{FQ}=0\right]\right)$;

STS split tensile strength:

UCS unconfined compressive strength;

$\mathrm{w}_{\mathrm{f}} \quad$ final water content (\%);

$\mathrm{w}_{\mathrm{L}} \quad$ Atterberg liquid limit (\%);

$\mathrm{W}_{\mathrm{P}} \quad$ Atterberg plastic limit (\%);

$\Delta \quad$ increment;

$\varepsilon_{\mathrm{ax}} \quad$ axial strain (\%);

$\gamma \quad$ unit weight $\left(\mathrm{kN} / \mathrm{m}^{3}\right)$;

\section{References}

Al-Tabbaa, A., Barker, P., Evans, C.W., 2009. Keynote lecture: design of deep mixing in infrastructure applications. In: International Symposium of Deep Mixing \& Admixture Stabilization. Okinawa, Japan.

ASTM., 1998. Standard Classification of Soils for Engineering Purposes (Unified Soil Classification System). D 2487, West Conshohoken, PA.

Cai, Y., Shi, B., Ng, C.W.W., Tang, C., 2006. Effect of polypropylene fibre and lime admixture on engineering properties of clayed soil. Eng. Geol. 87, 230-240.

Chauhan, M.S., Mittal, S., Mohanty, B., 2008. Performance evaluation of silty sand subgrade reinforced with fly ash and fibre. Geotext. Geomemb. 26 (5), 429-435.

Coelho, P.A.L.F., 2000. Geotechnical Characterization of Soft Soils. Study of the Experimental Site of Quinta do Foja. M.Sc. thesis. Department of Civil Engineering, University of Coimbra (in Portuguese).

Consoli, N.C., Vendruscolo, M.A., Fonini, A., Rosa, F.D., 2009. Fiber reinforcement effects on sand considering a wide cementation range. Geotext. Geomemb. 27 (3), 196-203.

Consoli, N.C., Bassani, M.A.A., Festugato, L., 2010. Effect of fiber-reinforcement on the strength of cemented soils. Geotext. Geomemb. 28 (4), 344-351.

Consoli, N.C., Zortéa, F., Souza, M., Festugato, L., 2011a. Studies on the dosage of fiber-reinforced cemented soils. J. Mater. Civ. Eng. 23 (12), 1624-1632.

Consoli, N.C., Moraes, R.R., Festugato, L., 2011b. Split tensile strength of monofilament polypropylene fiber-reinforced cemented sandy soils. Geosynth. Int. 18 (2), 57-62.

Consoli, N.C., Bellaver Corte, M., Festugato, L., 2012. Key parameter for tensile and compressive strength of fibre-reinforced soil-lime mixtures. Geosynth. Int. 19 (5), 409-414.

Consoli, N.C., Scapini, B., Festugato, L., 2013a. A practical methodology for the determination of failure envelopes of fiber-reinforced cemented sands. Geotext. Geomemb. 41, 50-54.

Consoli, N.C., Moraes, R.R., Festugato, L., 2013b. Parameters controlling tensile and compressive strength of fiber-reinforced cemented soil. J. Mater. Civ. Eng. 25 (10), 1568-1573.

Consoli, N.C., Moraes, R.R., Festugato, L., 2013c. Variables controlling strength of fibre-reinforced cemented soils. Ground Improv. 166 (4), 221-232.
Correia, A.A.S., 2011. Applicability of Deep Mixing Technique to the Soft Soil of Baixo Mondego. Ph.D. dissertation. University of Coimbra, Coimbra, Portugal (in Portuguese).

Custódio, D.G., 2013. Mechanical behaviour of a stabilized soft soil with polypropylene fibres. M.Sc. dissertation. University of Coimbra, Coimbra, Portugal (in Portuguese).

Edil, T.B., Staab, D.A., 2005. Practitioner's Guide for Deep-mixed Stabilization of Organic Soils and Peat. Final Rep. NDM302, National Deep Mixing Research Program. Federal Highway Administration, Washington, DC.

EN 197-1, 2000. Cement - Part 1: Composition, Specifications and Conformity Criteria for Common Cements. European Committee for Standardization, June 2010.

Estabragh, A.R., Namdar, P., Javadi, A.A., 2012. Behavior of cement-stabilized clay reinforced with nylon fiber. Geosynth. Int. 19 (1), 85-92.

Eurosoilstab, 2001. Development of Design and Construction Methods to Stabilise Soft Organic Soils. Design Guide Soft Soil Stabilization, p. 94. CT97-0351, EC Project No. BE 96-3177, Industrial \& Materials Technologies Programme (BriteEuRam III), European Commission.

Festugato, L., Fourie, A., Consoli, N.C., 2013. Cyclic shear response of fibre-reinforced cemented paste backfill. Géotech. Lett. 3, 5-12.

Hamidi, A., Hooresfand, M., 2013. Effect of fiber reinforcement on triaxial shear behavior of cement treated sand. Geotext. Geomemb. 36, 1-9.

Hernandez-Martinez, F.G., Sparrevik, M., Kvalvåg, ф., Eggen, A., Kvennås, A. Grini, R.S., 2009. Stabilization/solidification of two contaminate marine sediments. In: International Symposium of Deep Mixing \&Admixture Stabilization. Okinawa, Japan.

Hight, D.W., Jardine, R.J., Gens, A., 1987. "The Behaviour of Soft Clays". Embankments on Soft Clays. Special Publication. Bulletin of the Public Works Research Center, Athens.

Holm, G., 2005. Keynote Lecture: towards a sustainable society - recent advances in deep mixing. In: International Conference on Deep Mixing - Best Practice and Recent Advances: k13-k24. Swedish Deep Stabilization Centre, Stockholm, Sweden.

Janz, M., Johansson, S.-E., 2002. The Function of Different Binding Agents in Deep Stabilization. Swedish Deep Stabilization Research Centre, Report 9, Linköping, Sweden.

Kaniraj, S.R., Havanaji, V.J., 2001. Behavior of cement-stabilized fiber- reinforced fly ash-soil mixtures. J. Geotech. Geoenviron. Eng. 127 (7), 574-584.

Khattak, M.J., Alrashidi, M., 2006. Durability and mechanistic characteristics of fiber reinforced soil-cement mixtures. Int. J. Pavement Eng. 7 (1), 53-62.

Kitazume, M., Terashi, M., 2013. The Deep Mixing Method-Principle, Design and Construction. Coastal Development Institute of Technology. CRC Press/Balkema, Leiden, The Netherlands.

Kumar, A., Walia, B.S., Bajaaj, A., 2007. Influence of fly ash, lime and polyester fibers on compacted and strength properties of expansive soil. J. Material Civ. Eng. 19 (3), 242-248,

NP EN 1992-1-1, 2010. Eurocode 2 - Design of Concrete Structures. Part 1-1: General Rules and Rules for Buildings. Instituto Português da Qualidade, Lisbon, Portugal.

Olgun, M., 2013. Effects of polypropylene fiber inclusion on the strength and volume change characteristics of cement-fly ash stabilized clay soil. Geosynth. Int. 20 (4), 263-275.

Park, S., 2009. Effect of fiber reinforcement and distribution on unconfined compressive strength of fiber-reinforced cemented sand. Geotext. Geomemb. 27 (2), 162-166.

Park, S., 2011. Unconfined compressive strength and ductility of fiber-reinforced cemented sand. Constr. Build. Mater. 25 (2), 1134-1138.

Santos, A.P.S., Consoli, N.C., Heineck, K.S., Coop, M.R., 2010. High-pressure isotropic compression tests on fiber-reinforced cemented sand. J. Geotech. Geoenviron. Eng. 136 (6), 885-890.

Sukontasukkul, P., Jamsawang, P., 2012. Use of steel and polyprolylene fibers to improve flexural performance of deep soil-cement column. Constr. Build. Mater. 29 (1), 201-205.

Tang, C., Shi, B., Gao, W., Chen, F., Cai, Y., 2007. Strength and mechanical behaviour of short polypropylene fiber reinforced and cement stabilized clayed soil. Geotext. Geomemb. 25, 194-202.

Taylor, H.F.W., 1997. Cement Chemistry, second ed. Thomas Telford.

Terashi, M., 2005. Keynote lecture: design of deep mixing in infrastructure applications. In: International Conference on Deep Mixing - Best Practice and Recent Advances: k25-k45. Swedish Deep Stabilization Centre, Stockholm, Sweden.

Terashi, M., Kitazume, M., 2009. Keynote lecture: current practice and future perspective of QA/QC for deep-mixed ground. In: International Symposium of Deep Mixing \&Admixture Stabilization. Okinawa, Japan.

Venda Oliveira, P.J., Lemos, L.J.L., Coelho, P.A.L.P., 2010. Behavior of an atypical embankment on soft soil: field observations and numerical simulation. J. Geotech. Geoenviron. Eng. 136 (1), 35-47. 